\title{
Conversion of Spin into Directed Electric Current in Quantum Wells
}

\author{
S. D. Ganichev, ${ }^{1,2}$ E. L. Ivchenko, ${ }^{2}$ S. N. Danilov,${ }^{1}$ J. Eroms, ${ }^{1}$ W. Wegscheider, ${ }^{1, *}$ D. Weiss, ${ }^{1}$ and W. Prettl ${ }^{1}$ \\ ${ }^{1}$ Institut für Experimentelle und Angewandte Physik, Universität Regensburg, 93040 Regensburg, Germany \\ ${ }^{2}$ A. F. Ioffe Physico-Technical Institute of the RAS, 194021 St. Petersburg, Russia
}

(Received 31 January 2001)

\begin{abstract}
A nonequilibrium population of spin-up and spin-down states in quantum well structures has been achieved applying circularly polarized radiation. The spin polarization results in a directed motion of free carriers in the plane of a quantum well perpendicular to the direction of light propagation. Because of the spin selection rules the direction of the current is determined by the helicity of the light and can be reversed by switching the helicity from right to left handed. A microscopic model is presented which describes the origin of the photon helicity driven current. The model suggests that the system behaves as a battery which generates a spin polarized current.
\end{abstract}

DOI: $10.1103 /$ PhysRevLett.86.4358

The spin of electrons and holes in solid state systems is an intensively studied quantum mechanical property as it is the crucial ingredient for spintronics [1,2] and several schemes of quantum computation [3-5]. Among others, current investigations involve the spin lifetime in semiconductor devices [6-8] as well as the injection of spin polarized electrons (or holes) from semimagnetic semiconductor materials into semiconductors [9-11] or from ferromagnetic into nonmagnetic metals $[12,13]$.

It is well known that spin polarized electrons can be generated by circularly polarized light $[14,15]$ and, vice versa, that the recombination of spin polarized charged carriers results in the emission of circularly polarized light $[10,11,14]$. However, little is known about spin dependent photocurrents when a semiconductor is irradiated by circularly polarized light $[15,16]$. Helicity dependent photocurrents in semiconductors have been observed in bulk Te utilizing the peculiarities of the valence band structure ("camel back") at the first Brillouin zone boundary and in bulk GaAs subjected to an external magnetic field [15]. A first indication of such a photon helicity dependent photocurrent in semiconductor heterojunctions was found in recent far infrared experiments on $p$-type GaAs/AlGaAs heterojunctions containing a two-dimensional hole gas [17]. This preliminary experiment was discussed in phenomenological terms and lacked the microscopic connection to the carriers' spin.

The experiments on quantum wells (QWs) described below uncover a novel property of an unbalanced spin polarization: its ability to generate a directed current where the current's direction depends solely on the predominant spin orientation. This effect may be illustrated as an electron analog of mechanical systems where a rotational motion ("spin") is transmitted into a linear one ("current") like a rotating wheel on a hard surface. Below we point out that spin injection into quantum wells of zinc-blende-type material leads always to an electric current in the plane of the quantum well. The reduced dimensionality of quantum wells lowers the crystallographic symmetry and introduces $\boldsymbol{k}$-linear terms in the Hamiltonian. These $\boldsymbol{k}$-linear terms lift
PACS numbers: 73.50.Mx, 68.65.-k, 73.50.Pz, 78.30.Fs

the spin degenerate of energy bands in $\boldsymbol{k}$-space which, in the case of an unbalanced spin population, results in a current flow. The conversion of the carriers' spin polarization into an electric current has been observed in both $n$-type and $p$-type quantum wells as well as for different symmetry classes. The observed spin photocurrent flows in the quantum well perpendicular to the direction of the incident circularly polarized light. The current reverses its direction by switching the sign of helicity of the radiation and hence the spin orientation of free carriers. The effect is quite general and has been observed for all semiconductor systems investigated. The experimental data can be described by simple analytical expressions derived from a phenomenological theory. To close the gap between the phenomenological (spinless) theory and the spin of the carriers we present a microscopic model for absorption by both direct (interband and intersubband) and indirect intraband optical transitions.

The experiments were carried out on heterostructures belonging to two different classes of symmetry. Higher symmetric structures of the point group $D_{2 d}$ were (001)-MBE grown $n$-InAs/AlGaSb QWs with a $15 \mathrm{~nm}$ single InAs channel and (001)-MBE grown $n$-GaAs/AlGaAs single heterojunctions. Structures of the lower symmetry $C_{s}$ were (113) $A$-molecular-beam-epitaxy (MBE) grown $p$-GaAs/ AlGaAs single QWs and multiple QWs (MQWs) containing 20 wells of $15 \mathrm{~nm}$ width as well as $p$-GaAs/ AlGaAs MQWs with 400 wells of $20 \mathrm{~nm}$ width grown by metal-organic-chemical-vapor-deposition on vicinal (001) substrates. The growth direction of the latter structures was found to be tilted by an angle of $5^{\circ}$ with respect to the [001] crystallographic axis as has been verified by $\mathrm{x}$-ray diffraction. Samples of $n$ - and $p$-type with free-carrier densities between $10^{11}$ and $2 \times 10^{12} \mathrm{~cm}^{-2}$ were studied in the range from liquid helium to room temperature. Two pairs of Ohmic contacts have been centered along opposite sample edges (see insets of Figs. 1 and 2). For optical excitation we used a high power far infrared pulsed $\mathrm{NH}_{3}$ laser optically pumped by a transversely excited-atmospheric pressure $\mathrm{CO}_{2}$ laser which yields strong linearly polarized 


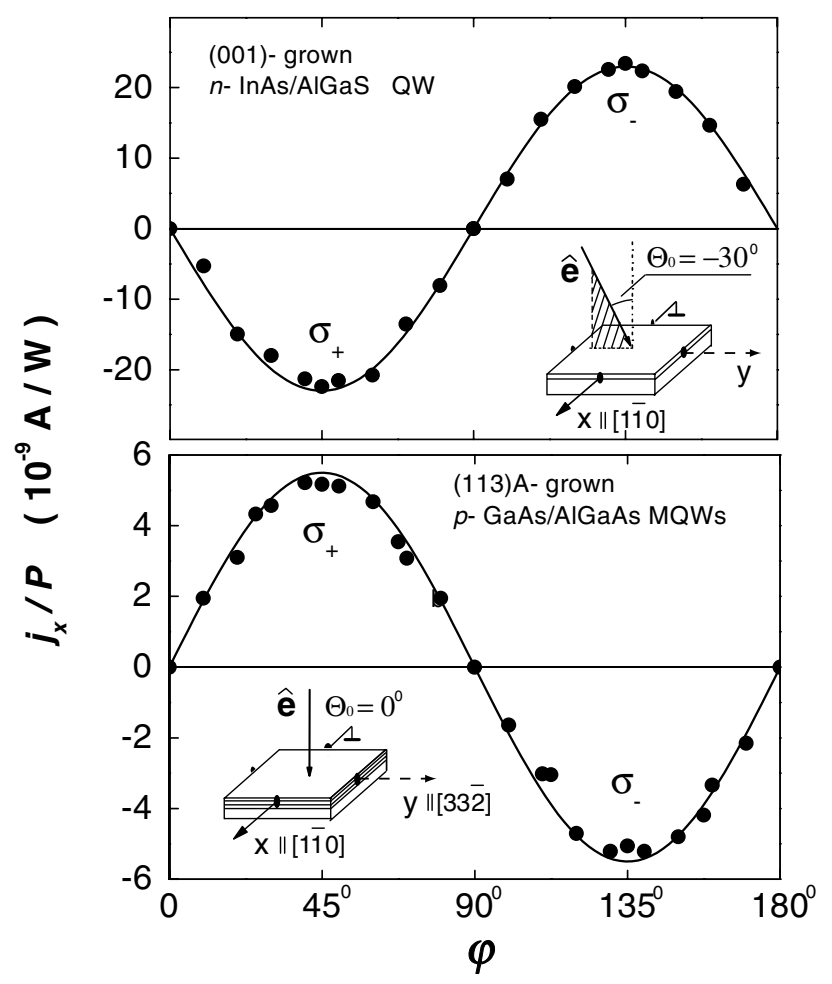

FIG. 1. Photocurrent in QWs normalized by the light power $P$ as a function of the phase angle $\varphi$ defining helicity. Measurements are presented for $T=300 \mathrm{~K}$ and $\lambda=76 \mu \mathrm{m}$. The insets show the geometry of the experiment. Upper panel: oblique incidence of radiation with an angle of incidence $\Theta_{0}=-30^{\circ}$ on $n$-type (001)-grown InAs/AlGaSb QWs (symmetry class $D_{2 d}$ ). The current $j_{x}$ is perpendicular to the direction of light propagation. Lower panel: normal incidence of radiation on $p$-type (113) $A$-grown GaAs/AlGaAs QWs (symmetry class $C_{s}$ ). The current $j_{x}$ flows along the [110] direction perpendicular to the mirror plane of the $C_{s}$ symmetry. Full lines are fitted using one parameter according to Eqs. (1) and (2).

emission at wavelengths $\lambda$ between 35 and $280 \mu \mathrm{m}$ corresponding to photon energies ranging from 55 to $6 \mathrm{meV}$ with power $P$ up to $100 \mathrm{~kW}$ [18]. The radiation induces indirect (Drude-like) optical transitions in the lowest conduction subband of our $n$-type samples and direct optical transitions between valence subbands (heavy hole-light hole) in the $p$-type samples. Crystalline quartz $\lambda / 4$ plates allowed us to modify the laser light polarization from linear to circular. The helicity $P_{\text {circ }}$ of the incident light varied from -1 (left handed circular, $\sigma_{-}$) to +1 (right handed circular, $\left.\sigma_{+}\right)$according to $P_{\text {circ }}=\sin 2 \varphi$ where $\varphi$ is the angle between the initial polarization plane and the optical axis of the $\lambda / 4$ plate.

The current $j_{x}$ generated by the circularly polarized light in the unbiased devices was measured via the voltage drop across a $50 \Omega$ load resistor in a closed circuit configuration. The voltage was measured with a storage oscilloscope. The measured current pulses of $100 \mathrm{~ns}$ duration reflect the corresponding laser pulses. In Figs. 1 and 2 we present measurements carried out at room temperature for (001)- $n$-InAs and (113) $A$ - $p$-GaAs/AlGaAs quantum wells

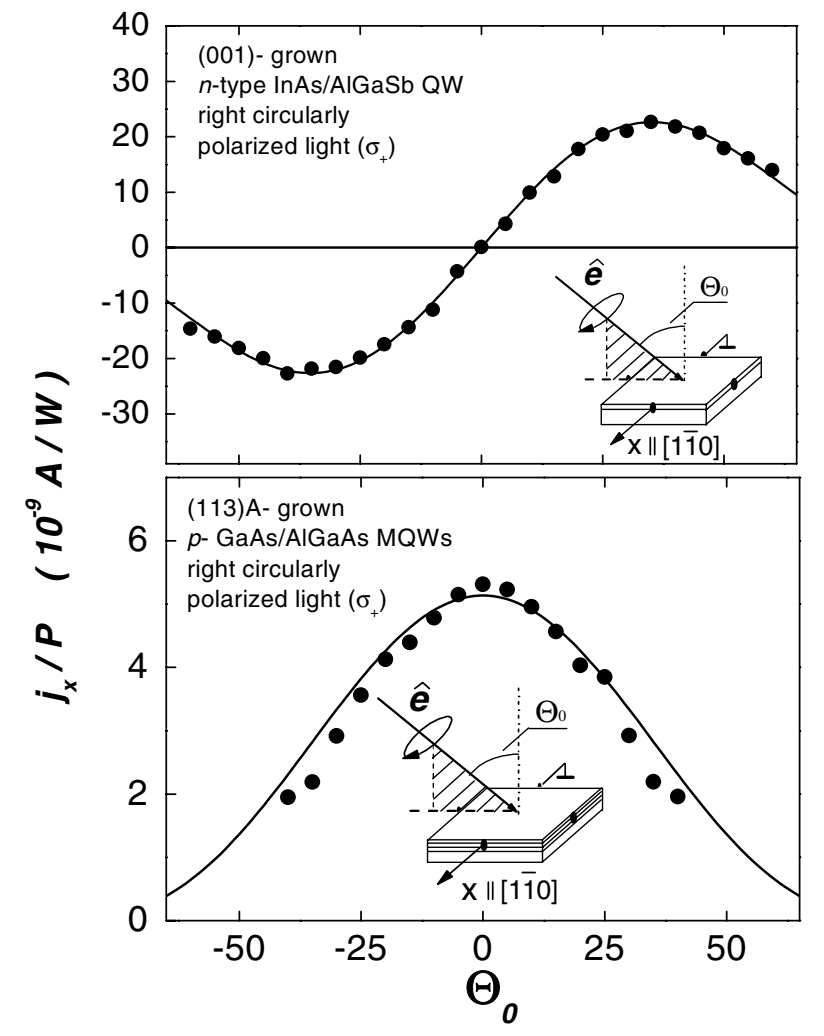

FIG. 2. Photocurrent in QWs normalized by the light power $P$ as a function of the angle of incidence $\Theta_{0}$ for right circularly polarized radiation $\sigma_{+}$measured perpendicular to light propagation $(T=300 \mathrm{~K}, \lambda=76 \mu \mathrm{m})$. Upper panel: $n$-type (001)-grown InAs/AlGaSb QWs $\left(D_{2 d}\right)$. Lower panel: $p$-type (113) $A$-grown $\mathrm{GaAs} / \mathrm{AlGaAs} \mathrm{QWs}\left(C_{s}\right)$. Full lines are fitted using one parameter according to Eqs. (1) and (2).

as representatives of the $D_{2 d}$ and $C_{s}$ symmetry classes, respectively.

In (001)-oriented samples of the higher symmetry class $D_{2 d}$ a signal proportional to the helicity $P_{\text {circ }}$ is observed only under oblique incidence and the photocurrent is perpendicular to the wave vector of the incident light. The reversal of the current direction when the polarization switches from left-handed to right-handed circular is clearly seen in the upper panel of Fig. 1. In samples of the lower symmetry class $C_{s}$ grown on a (113)-GaAs surface, the spin photocurrent can be observed also under normal incidence as shown in the lower panel of Fig. 1. The same result is obtained for the $p$-type QWs grown on the vicinal (001)-substrate. Because of the misalignment the symmetry class is again $C_{s}$. The current flows always along the [110] direction perpendicular to the plane of mirror reflection of the point group $C_{s}$, independent of the incidence plane of the laser beam. In Fig. 2 we take a closer look at the dependence of the photocurrent on the angle of incidence $\Theta_{0}$ of the circularly polarized laser beam. For (001)-oriented samples $\left(D_{2 d}\right.$ symmetry), a variation of $\Theta_{0}$ in the plane of incidence normal to $x$ changes the sign of the current $j_{x}$ for normal incidence, $\Theta_{0}=0$, as can be seen in the upper panel of Fig. 2. The lower panel of 
Fig. 2 displays the angular dependence for (113)-oriented quantum wells ( $C_{s}$ symmetry). A photocurrent proportional to the helicity $P_{\text {circ }}$ was observed in all samples for all wavelengths and temperatures investigated. While we have also observed photovoltages across the other pairs of contacts, these voltages do not change sign upon the change of helicity. They are ascribed to the linear photogalvanic effect [15] and the photon drag effect [19] which are outside the scope of the present investigation.

The solid lines in Figs. 1 and 2 are obtained from a phenomenological picture outlined below which perfectly describes the experimental observations. In this picture, the connection between the photocurrent, $\boldsymbol{j}$, sensitive to the helicity of light and the incoming light is given by the secondrank pseudotensor $\boldsymbol{\gamma}$ as $\boldsymbol{j}=\boldsymbol{\gamma} \cdot \hat{\boldsymbol{e}} E^{2} P_{\text {circ }}$ where $E$ is the amplitude of the electric field of the light and $\hat{\boldsymbol{e}}$ is the unit vector pointing in the direction of the light propagation [17]. The pseudotensor $\boldsymbol{\gamma}$ can have nonzero components in gyrotropic systems which comprise quantum wells prepared on zinc-blende structures materials. For the higher symmetry $D_{2 d}$ valid for quantum well structures grown along the principal axis [001], a photocurrent can be generated only under oblique incidence of irradiation. For the point group $D_{2 d}$ the nonzero components of $\gamma$ are $\gamma_{x y}=\gamma_{y x}$ with $x\|[1 \overline{1} 0], y\|[110]$. If $(y, z)$ is the plane of incidence, then the photocurrent is induced along $x$ with

$$
j_{x}=\gamma_{x y} t_{p} t_{s} \sin \Theta E_{0}^{2} P_{\text {circ }},
$$

where $E_{0}$ is the electric field amplitude in vacuum, $t_{p}$ and $t_{s}$ are transmission coefficients after Fresnel's formula for linear $p$ and $s$ polarizations [15], $\Theta$ is the angle of refraction defined by $\sin \Theta=\sin \Theta_{0} / n$, and $n$ is the index of refraction. In the low-symmetry QWs grown along $z \|[h h l]$ with $[h h l] \neq[001]$ or [111], the point group is $C_{s}$ which contains only two elements, the identity and one mirror reflection plane normal to the [110] direction. In this case additionally the pseudotensor component $\gamma_{x z}$ is nonzero allowing the generation of a photocurrent along $x \|$ [110] also under normal incidence of radiation. The corresponding contribution to the photocurrent is

$$
j_{x}=\gamma_{x z} t_{p} t_{s} \cos \Theta E_{0}^{2} P_{\text {circ }} .
$$

The currents measured along $x$ as a function of the phase angle $\varphi$ (Fig. 1) and the angle of incidence $\Theta_{0}$ (Fig. 2) are in a very good agreement with the phenomenological expressions Eq. (1) for symmetry $D_{2 d}$ and Eq. (2) for $C_{s}$. Both figures show experimental data compared to calculations which were fitted with one ordinate scaling parameter.

Microscopically a conversion of photon helicity into a spin photocurrent arises due to $\boldsymbol{k}$-linear terms in the effective Hamiltonian $H^{(1)}=\beta_{l m} \sigma_{l} k_{m}$ where $\boldsymbol{k}$ is the electron wave vector, $\sigma_{l}$ are the Pauli spin matrices, and $\beta_{l m}$ are real coefficients. The coefficients $\beta_{l m}$ form a pseudotensor subjected to the same symmetry restriction as the pseudotensor $\boldsymbol{\gamma}$. The coupling between $\sigma_{l}$ and the wave vector of the charged carriers $k_{m}$ as well as spin controlled selection rules, described below, yield a net current dependent upon circularly polarized optical excitation. The effect is most easily conceivable for both $n$ - and $p$-type materials from the schematic band structure shown in Fig. 3. For the sake of simplicity we assume a band structure (twodimensional dispersion) consisting only of the lowest conduction subband $e 1$ and the highest heavy hole subband $h h 1$. The $\boldsymbol{k}$-linear terms are taken into account only for the valence band and result in a splitting into two parabolas of different spin. In the case of $C_{s}$ symmetry the splitting is given by $E_{h h 1, \pm 3 / 2}(\boldsymbol{k})=-\left(\hbar^{2} k^{2} / 2 m_{h}\right) \pm \beta k_{x}$ [20].

First we consider direct optical transitions which, depending on the photon energy, may be of interband or intersubband type (heavy hole-light hole or heavy hole-heavy hole subbands in $p$-type materials). The theoretical concept of the model is the same for both types of transitions. However, for intersubband transitions the $\boldsymbol{k}$-linear terms, which are different for different bands and subbands, have to be taken into account for both subbands involved and complicate the picture. Hence, we concentrate on the interband excitations shown in Fig. 3a. While not directly illustrating the transitions in our $p$-type QWs, Fig. 3a makes the point more transparent than a discussion of the more complex intersubband transitions in the valence band. Qualitatively the results are the same. In Fig. 3a the allowed optical transitions are from $s=-3 / 2$ to $s=-1 / 2$
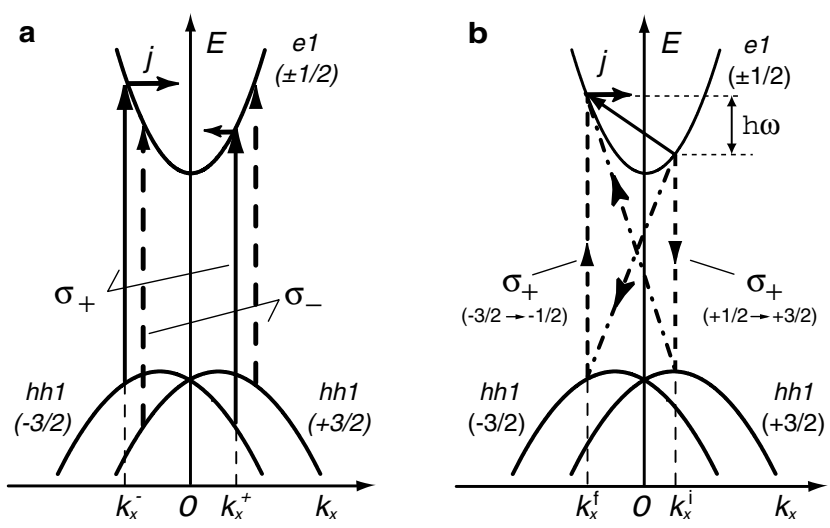

FIG. 3. Microscopic picture describing the origin of spin polarized photocurrents. The essential ingredient is the splitting of the valence band due to $\boldsymbol{k}$-linear terms. In (a) $\sigma_{+}$excitation induces direct transitions (solid arrows) from $s=-3 / 2(h h 1)$ to $s=-1 / 2(e 1)$ with unbalanced occupation of the positive $k_{x}$ and negative $k_{x}$ states resulting in a spin polarized photocurrent. For $\sigma_{-}$excitation (dashed arrows) both the spin orientation of the charge carriers and the current direction get reversed. (b) A free electron transition (solid arrow) in the conduction band $e 1$ via intermediate states in the valence subbands. Two representative virtual transitions for $\sigma_{+}$excitation are illustrated. One is an optical transition from $s=+1 / 2$ to $s=+3 / 2$ (dashed line, downward arrow) and a transition involving a phonon from $s=+3 / 2$ back to the conduction band (dash-dotted line, upward arrow). The other is a phonon transition from the conduction band to the $s=-3 / 2$ intermediate state in $h h 1$ and an optical transition from $s=-3 / 2$ to $s=-1 / 2$. While the first route depopulates preferentially initial states of spin $+1 / 2$ for $k_{x}^{i}>0$, the second one populates preferentially the final state of $s=-1 / 2$ states for $k_{x}^{f}<0$. This together with the unbalanced occupation of the $k$ space results in a spin polarized photocurrent. 
for the $\sigma_{+}$photon polarization and from $s=3 / 2$ to $s=1 / 2$ for the $\sigma$ - polarization. The $s$ are the spin quantum numbers of the corresponding electron states. Under circularly polarized radiation with a photon energy $\hbar \omega$ and for fixed value of $k_{y}$ energy and momentum conservation allow transitions only for two values of $k_{x}$. For the $\sigma_{+}$ photons these $k_{x}$ values of photogenerated electrons are

$k_{x}^{ \pm}=-\frac{\mu}{\hbar^{2}} \beta \pm\left[\frac{2 \mu}{\hbar^{2}}\left(\hbar \omega-E_{g}\right)-k_{y}^{2}+\left(\frac{\mu}{\hbar^{2}} \beta\right)^{2}\right]^{-1 / 2}$,

where $E_{g}$ is the energy gap, $m_{e, h}$ are the effective masses of electrons and holes, respectively, and $\mu$ is the reduced electron-hole effective mass $m_{e} m_{h} /\left(m_{e}+m_{h}\right)$. The corresponding transitions are shown in Fig. $3 a$ by the solid vertical arrows with their center of "mass" shifted from the point $k_{x}=0$ by $-\beta\left(\mu / \hbar^{2}\right)$. Thus the sum of the electron velocities in the excited states, $\hbar\left(k_{x}^{+}+k_{x}^{-}\right) / m_{e}=$ $-2\left(\mu / m_{e}\right)(\beta / \hbar)$, is nonzero and the contribution of $k_{x}^{ \pm}$ photoelectrons to the current do not cancel as in the case $\beta=0$. Consequently, a spin polarized net current in the $x$ direction results. Changing the photon helicity from +1 to -1 inverts the current because the "center of mass" of these transitions is now shifted to $+\beta\left(\mu / \hbar^{2}\right)$ (see dashed arrows in Fig. 3a).

Now we consider indirect transitions. This situation is realized in our experiments on $n$-type QWs, where the photon energy is not high enough to excite direct intersubband transitions. Because of energy and momentum conservation intraband transitions can occur only by absorption of a photon and simultaneous absorption or emission of a phonon. This process is described by virtual transitions involving intermediate states. Transitions via intermediate states within one and the same subband do not contribute to the spin photocurrent. However, spin selective indirect optical transitions excited by circularly polarized light with both initial and final states in the conduction band can generate a spin current if virtual processes involve intermediate states in different subbands. Figure $3 b$ sketches the underlying mechanism for $\sigma_{+}$polarization. The two virtual transitions shown represent excitations which, for $\sigma_{+}$ helicity, "transfer" electrons from states with positive $k_{x}$ to states with negative $k_{x}$. The transfer results in a redistribution of the spins. This imbalance of occupied $k$ states leads to a spin polarized current in the $x$ direction. Switching the helicity from $\sigma_{+}$to $\sigma_{-}$reverses the process and results in a spin current in the opposite direction.

The picture of the spin photocurrent given so far involved the asymmetry of the momentum distribution of carriers. For a fully quantitative description of the spin polarized net current a possible asymmetry of the spin flip scattering rate of the carriers has to be included [16] which determines the decay of the photocurrent after pulsed excitation. In the present model of a spin degenerate conduction subband the decay is solely governed by momentum relaxation. Taking a spin split subband into account, the decay of the photocurrent is determined by both spin and momentum relaxation times where the spin relaxation can be much slower than momentum relaxation.

In summary, the experiments carried out on different types of quantum wells have shown that circularly polarized light can generate a directed electric current even at room temperature. The microscopic picture given above requires that the generated current is spin polarized and suggests that the system can be considered as a source for spin polarized currents. We emphasize that in gyrotropic media with $\boldsymbol{k}$-linear terms in the Hamiltonian, spin injection yielding an imbalance of spin orientation leads always to a current. As quantum wells based on III-V and II-VI compounds are gyrotropic, spin polarization causes in any case a current flow. The effect provides an easy access to investigate spin phenomena in low dimensional semiconductors.

The high quality InAs quantum wells were kindly provided by J. De Boeck and G. Borghs from IMEC Belgium. The technical support of M. Bichler is gratefully acknowledged. We also acknowledge financial support from the DFG, the RFFI, and the NATO linkage program.

*Also at Walter Schottky Institut, TU München, 85748 Garching, Germany.

[1] S. Datta and B. Das, Appl. Phys. Lett. 56, 665 (1990).

[2] G. A. Prinz, Phys. Today 48, No. 4, 58 (1995).

[3] B.E. Kane, Nature (London) 393, 133 (1998).

[4] D. Loss and D. P. DiVincenzo, Phys. Rev. A 57, 120 (1998).

[5] D. P. DiVincenzo, D. Bacon, J. Kempe, G. Burkard, and K. B. Whaley, Nature (London) 408, 339 (2000).

[6] J. M. Kikkawa, I. P. Smorchkova, N. Samarth, and D. D. Awschalom, Science 227, 1284 (1997).

[7] D. Hägele, M. Oestreich, W. W. Rühle, N. Nestle, and K. Eberl, Appl. Phys. Lett. 73, 1580 (1998).

[8] J. M. Kikkawa and D. D. Awschalom, Nature (London) 397, 139 (1999).

[9] M. Oestreich et al., Appl. Phys. Lett. 74, 1251 (1999).

[10] R. Fiederling et al., Nature (London) 402, 787 (1999).

[11] Y. Ohno et al., Nature (London) 402, 790 (1999).

[12] M. Johnson and R. H. Silsbee, Phys. Rev. Lett. 55, 1790 (1985).

[13] F. J. Jedema, A. T. Filip, and B. J. van Wees, Nature (London) 410, 345 (2001).

[14] Optical Orientation, edited by F. Meier and B. P. Zakharchenya (Elsevier Science Publishing, Amsterdam, 1984).

[15] E. L. Ivchenko and G. E. Pikus, Superlattices and Other Heterostructures. Symmetry and Optical Phenomena (Springer, Berlin, 1997).

[16] E. L. Ivchenko, Yu. B. Lyanda-Geller, and G. E. Pikus, Sov. Phys. JETP 71, 550 (1990).

[17] S. D. Ganichev, H. Ketterl, W. Prettl, E. L. Ivchenko, and L. E. Vorobjev, Appl. Phys. Lett. 77, 3146 (2000).

[18] S.D. Ganichev, Physica (Amsterdam) 273-274B, 737 (1999).

[19] S. D. Ganichev, S. A. Emel'yanov, and I. D. Yaroshetskii, JETP Lett. 35, 368 (1982).

[20] The case of the $D_{2 d}$ symmetry can be considered in a similar way. For the $C_{s}$ symmetry described here the coordinate system is $z\|[h h l], x\|[1 \overline{1} 0], y \|[l l(2 \bar{h})]$. 